\title{
RESEARCH ARTICLE \\ Influence of Amino acids on the Economic Characters of Silkworm, Bombyx mori L.
}

\author{
Murugesh K A*, Aruna R and Chozhan K \\ Department of Sericulture, Forest College \& Research Institute,Tamil Nadu Agricultural University, Mettupalayam - 641301
}

\begin{abstract}
Studies were undertaken to assess the influence of amino acids on the larval and cocoon characters of silkworm, Bombyx mori L. For the experiments, three amino acids namely glycine, alanine and serine were used at various concentrations viz., 10, 20, 50, 100 and 200 ppm. Supplementation of amino acids to silkworm larvae was done once daily from the first day of the fifth instar to spinning. The results revealed that all the concentrations positively influenced the mature larval weight, effective rate of rearing, effective rate of rearing, cocoon weight, shell weight, shell ratio and filament length. However, the improvement in all the parameters studied was highest at the concentration of 10 ppm, 100 ppm and 100 ppm, respectively in glycine, alanine and serine, over all other concentrations tested. Per os application of amino acids at specific concentrations also significantly reduced the fifth instar larval duration, a desirable character.
\end{abstract}

Key words: Bombyx mori; Cocoon weight; Shell weight; ERR; Amino acids

\section{INTRODUCTION}

Mulberry silkworm, Bombyx mori L. emits the lustrous raw material for the production of 'Queen of the Textiles', the silk, which could not be substantiated even by the advanced scientific innovations. B. mori is a domesticated oligophagus sericigenous insect that feeds only on mulberry, Morus spp. Mulberry leaves supply all the essential nutrients needed for silk protein synthesis, such as amino acids, minerals, sugars, proteins, vitamins, etc. However, the nutritional status of the mulberry varies based on the prevailing environmental conditions and crop management practices. Manya-times, due to the poor nutrient status of mulberry, crop failure occurs, leading to substantial economic loss to the silkworm growers. This could be avoided by supplying the essential nutrients required for the normal growth and development of silkworm larvae in sufficient quantities through the fortification of mulberry leaves.

Among different supplementation experiments with vitamins, antibiotics, amino acids, sugars, trace elements, etc., the fortification of mulberry leaves with amino acids was found to be more beneficial since it improved the leaf quality and the silk yield by 40-60 per cent (Senguptha et al., 1972).

The amino acids play an essential role in different phases of insect life, and their overall metabolic pathways are the same in insects as in other animal classes. The amino acids fulfill additional metabolic functions such as osmoregulation in aquatic insects (Beadle and Shaw. 1950), neural transmitters (Chen and Widmer, 1968), detoxification of specific metabolites, synthesis of phospholipids, energy source and morphogenetic processes. The major function of intracellular amino acid is assumed to be an obligatory precursor for protein synthesis. High levels of free amino acids characterize insects, their total haemolymph quantity is about 100 to 300 times higher than that present in the human blood (Chen and Widmer, 1968).

Studies undertaken by various researchers over the period of time on supplementation with amino acids showed significant enhancement in the production of raw silk. Enrichment of mulberry leaves with different amino acids viz., glycine (Sharadha and Bhat, 1956; Ravi et al., 1994 and Saad et al., 2019), essential amino acids (Arai and Ito, 1964; Ito, 1967 and Inokuchi et al., 1967), tyrosine, phenylalanine and alanine (Nagarajan and Radha, 1990), glycine, serine and alanine (Mustafa and Elkaraksy, 1990), aspatic acid (Kabila et al., 1994), glycine, phenylalanine, serine and aspartic acid (Vadivel, 1995), alanine and asparginine (Radjabi et al., 2010), arginine and histidine (Chakrabarthy and Kaliwal, 2012) and serine (Gokul, 2015) has increased the larval, cocoon, reeling and silk productivity-related parameters.

The findings of different workers motivated the authors to undertake investigations to ascertain 
the exact minimal concentration of selected amino acids required for increasing the larval and cocoon characters. Hence, an attempt has been made in this direction with the amino acids namely glycine, alanine and serine.

\section{MATERIALS AND METHODS}

The experiements were conducted by fortifying the mulberry leaves with amino acids such as glycine, alanine and serine individually to ascertain the influences of amino acids in the economic characters of silkworm. The methodology adopted and materials used for the experiments are detailed below.

\section{i) Disinfection}

Silkworm rearing house and rearing equipment were thoroughly disinfected with 2.5 per cent Serichlor in 0.5 per cent slaked lime solution before commencement of silkworm rearing. Subsequently, the rearing house was closed in airtight condition for one day to ensure 100 per cent disinfection and then opened to remove the chlorine smell (Dandin and Giridhar, 2014).

\section{ii) Silkworm rearing}

The silkworm, Double Hybrid (DH), was used for experiments and the rearing was conducted during the period favorable for bivoltine silkworm i.e., August to February. The leaves from well grown mulberry plants (Variety V1) were utilized for silkworm rearing. The feeding for larvae was provided three times per day without starvation. The bed cleaning was done using nylon net and the sufficient spacing was provided as age of the larvae increased. The bed disinfectant was dusted at $4-5 \mathrm{~g} / \mathrm{sq}$. $\mathrm{ft}$ area to prevent diseases immediately after moulting and the leaves were provided 45 - 60 minutes later (Dandin and Giridhar, 2014). The ripened larvae were mounted with Rotary mountage and well-built cocoons were harvested six days after the spinning.

\section{iii) Administration of amino acids}

The stock solution of 500 ppm was prepared by completely dissolving $500 \mathrm{mg}$ of amino acid in one litre of distilled water. Various required concentrations viz., 10, 20, 50, 100 and 200 ppm were prepared carefully by serial dilution technique from the stock solution.

The fresh mulberry leaves were harvested on 60 days after pruning and weighed with an electronic balance. The weighed quantity of leaves was sprayed with an aqueous solution of the respective amino acid by using a glass atomizer. Two larval groups, one fed with mulberry leaves sprayed with distilled water and another fed with untreated mulberry leaves (Control), was maintained for comparison.
The treated leaves were shade dried for 15 to 30 minutes and fed to silkworm larvae once daily in the morning from the first day of the fifth instar to spinning.

\section{iv) Observations recorded}

Various economic characters viz., mature larval weight, fifth instar larval duration, effective rate of rearing (ERR), cocoon weight, shell weight, shell ratio, and filament length were observed. For recording the data, randomly selected 10 samples (larvae, cocoon and shell) were used from the experiments.

\section{Statistical analysis}

The experiments were conducted by taking up silkworm rearing two times in Completely Randomized Design. Each treatment was replicated three times with 50 larvae per replication. Statistical analysis of data was done using the method suggested by Panse and Sukhatme (1957). The results of the experiments are presented below.

\section{RESULTS AND DISCUSSION}

Per os administration of amino acids such as glycine, alanine and serine in various concentrations viz., 10, 20, 50, 100 and 200 ppm to B. mori larvae once daily in the morning starting from first day of fifth instar to spinning significantly increased the larval as well as cocoon economic characters. The results of the experimental studies undertaken are given below elaborately.

\section{Influence of Glycine on larval and cocoon characters}

\section{a. Larval characters}

The studies showed that the enrichment of mulberry leaves with glycine in varied concentrations elucidated positive effects on the biological characters of silkworm larvae (Fig. 1). Per os administration of glycine @ 10 ppm significantly improved the mature larval weight to $4.18 \mathrm{~g}$ from $3.37 \mathrm{~g}$ which was statistically superior over all other concentrations followed by $20 \mathrm{ppm}$ (4.03 g). The concentrations such as 50 and 100 ppm were found to be statistically on par with each other. Among the different concentrations tested, $200 \mathrm{ppm}$ recorded minimum mature larval weight of $3.68 \mathrm{~g}$; however, this was found to be statistically higher than the control. This result is in accordance with the findings of Sridhar and Radha (1986), who reported the highest larval weight gain at $10 \mathrm{ppm}$ level of glycine. Saad et al. (2019) observed that the feeding the $B$. mori larvae with mulberry leaves immersed in 1 per cent glycine during fifth instar once resulted in significant increase in mature larval weight (5.12 g) over the control (4.74 g). This finding also falls in line with the present observation. 


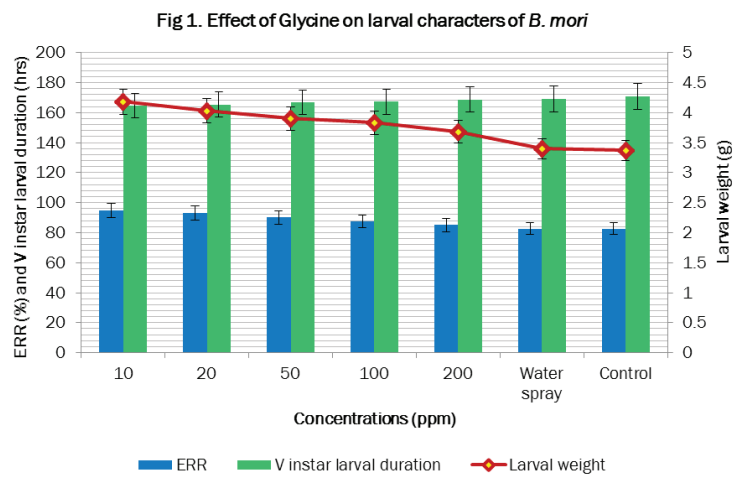

The effective rate of rearing (ERR) is the main indicator for assessing successful silkworm rearing. The present experiments proved that glycine @ 10 ppm was the best treatment in ERR (95.00\%), among the various concentrations studied, followed by 20 ppm (92.95\%). The concentrations namely 50 and 100 ppm registered ERR of 90.10 and 87.50 per cent, respectively and were found to be statistically on par with each other. The minimum ERR of 82.70 per cent was recorded in the control. Here, the enhancement in ERR ranged from 2.50 to 9.38 per cent. The present result is in parallel with the findings of Ito and Arai (1965) and Chakrabarthy and Kaliwal, (2012), who reported that higher ERR indirectly indicates the lesser susceptibility of the silkworm larvae to dreaded diseases and this resistance was due to conversion of amino acids into antibodies.

The reduction in larval duration is beneficial since it reduces the labour requirement for feeding the silkworm. In the present experiment, the fifth instar larval duration was significantly brought down to $164.50 \mathrm{hrs}$ from $170.60 \mathrm{hrs}$ due to the application of glycine at $10 \mathrm{ppm}$ concentration. This was on par with 200 ppm and significantly superior over all other concentrations tested. The larval batch fed with 50, 100 and 200 ppm treated mulberry leaves did not differ statistically with each other. This result fall in line with Ito and Arai, (1965), Mustafa and Elkaraksy (1990), Vadivel (1995) and Saad et al. (2019) who reported that supplementation of glycine significantly decreased the fifth instar larval duration.

\section{b. Cocoon characters}

Investigations obviously proved that fortification of mulberry at varied concentrations of glycine and feeding the silkworm larvae had significant impact on the cocoon characters (Table 1). Maximum cocoon weight of $2.10 \mathrm{~g}$ was registered in the larval batch fed with $10 \mathrm{ppm}$ of glycine sprayed mulberry leaves, which was followed by 20 ppm (2.03 g). The treatments, 50 and 100 ppm, were statistically on par by registering the cocoon weight of 1.96 and $1.93 \mathrm{~g}$, respectively. Among the different concentrations of glycine, the lower cocoon weight of $1.85 \mathrm{~g}$ was registered in $200 \mathrm{ppm}$, however, this treatment showed improvement statistically over the control $(1.77 \mathrm{~g})$. The present observation is in parity with the findings of Murthy and Srinivasaya (1953), who reported that supplementation of glycine, increased the cocoon weight by enhancing the sericin and fibroin synthesis in the posterior and middle silk gland respectively. The report by Saad et al. (2019) revealed that feeding the silkworm during fifth instar once with mulberry leaves treated with 1 per cent glycine significantly enhanced cocoon weight to $1.64 \mathrm{~g}$ from $0.956 \mathrm{~g}$ in control, also corroborates the present experimental results.

Shell weight and shell ratio are important characters that denote the available silk filament in a cocoon, based on which the floor price of the cocoon is arrived. In the present study, the shell weight and shell ratio of $0.46 \mathrm{~g}$ and 21.90 per cent, respectively, were recorded in the larval batch treated with glycine @ 10 ppm. The treatment with 50 ppm concentration was found to be statistically on par with 20 and 100 ppm in the case of shell weight and shell ratio. The lowest shell weight and shell ratio of 0.29 and 16.38 per cent, respectively, were registered in the control. This result corroborates with the previous findings of Krishnappa (1987), Kabila et al. (1994), Ravi et al. (1994) and Vadivel (1995), who have reported that application of glycine at 10 ppm either individually or in combination increased the shell weight and shell ratio.

Table 1. Effect of glycine on cocoon characters of B. mori

\begin{tabular}{lcccc}
\hline $\begin{array}{c}\text { Concentration } \\
(\mathbf{p p m})\end{array}$ & $\begin{array}{c}\text { Cocoon } \\
\text { weight } \mathbf{( g )}\end{array}$ & $\begin{array}{c}\text { Shell } \\
\text { weight }(\mathbf{g})\end{array}$ & $\begin{array}{c}\text { Shell } \\
\text { ratio (\%) }\end{array}$ & $\begin{array}{c}\text { Filament } \\
\text { length }(\mathbf{m})\end{array}$ \\
\hline 10 & 2.10 & 0.46 & 21.90 & 1096 \\
20 & 2.03 & 0.42 & 20.68 & 1075 \\
50 & 1.96 & 0.38 & 19.38 & 1052 \\
100 & 1.93 & 0.36 & 18.65 & 1033 \\
200 & 1.85 & 0.32 & 17.30 & 1027 \\
Water spray & 1.77 & 0.29 & 16.38 & 1006 \\
Control & 1.77 & 0.29 & 16.38 & 1002 \\
SEd & 0.03 & 0.01 & 0.60 & 8.60 \\
CD (P=0.05) & 0.06 & 0.03 & 1.20 & 17.00 \\
\hline
\end{tabular}

Values are mean of three replications and pooled mean of two rearing.

The application of glycine to silkworm larvae enhanced the length of silk filament. Significantly highest filament length of $1096 \mathrm{~m}$ was recorded in the larval batch fed with mulberry leaves having 10 ppm glycine. The next better concentrations were in the order of 20,50, 100 and 200 ppm. The lowest filament length of $1002 \mathrm{~m}$ was observed in the control. Here, the improvement in the filament length ranged from 2.50 to 9.38 per cent. Fibroin content directly affects filament length, which might be largely influenced by lower concentration of glycine. The present observation is in line with the findings of Kodie et al. (1965), who observed that incorporating glycine residue into the fibroin leads to an increase 
in filament length. Further, the reports over the period of time by various researchers (Sengupta et al., 1972; Sridhar and Radha, 1986 and Krishnapa, 1987) showing enhanced filament length due to the application of glycine also confirm the present observations. In addition to this, the finding by Saad et al. (2019), who reported that feeding the fifth instar worms with mulberry leaves treated with 1 per cent glycine, elucidated a significant increase in the length of silk filament from $1226.25 \mathrm{~m}$ (control) to $1466.44 \mathrm{~m}$, also falls in line with the experimental results.

\section{Influence of alanine on larval and cocoon characters}

\section{a. Larval characters}

Supplementation of amino acid with mulberry leaves invariably increased the larval characters of silkworm over the control (Fig. 2). The application of alanine @ 100 ppm significantly increased the larval weight (4.08 g) than all other concentrations. The treatments having 200 and 50 ppm were found to be on par with each other with the cocoon weight of 3.87 and $3.82 \mathrm{~g}$, respectively. These concentrations were followed by 20 and $10 \mathrm{ppm}$. The lowest mature larval weight of $3.23 \mathrm{~g}$ was registered in the control. This result is very well in line with the findings of Sengupta et al. (1972), Mustafa and Elkarasky (1990) and Ravi et al. (1994), who reported an increase in mature larval weight by supplementation of amino acid mixture. Radjabi (2010) observed that fortification of mulberry leaves with 0.1 per cent alanine and feeding the silkworm larvae from the beginning of the fourth instar to the end of the fifth instar, once in a day significantly improved the larval weight to $2.91 \mathrm{~g}$ from $2.58 \mathrm{~g}$ in control. This finding also supports the present observations.

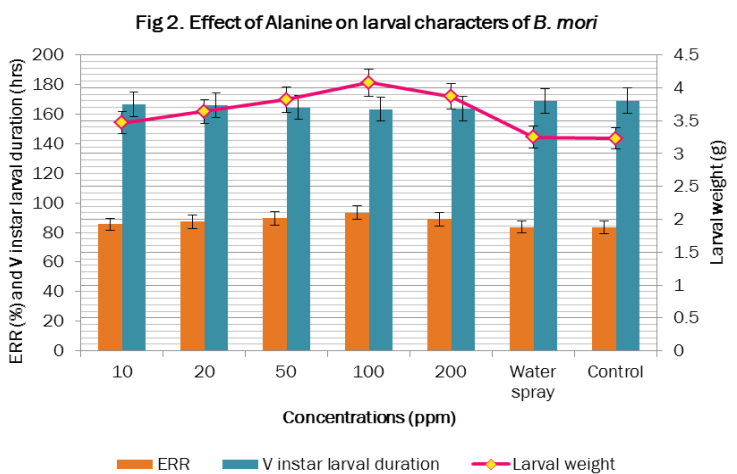

Significantly superior ERR of 93.40 per cent was recorded in alanine @ 100 ppm over the control. The next better treatments, 200 and 50 ppm were found to be on par with each other, which were followed by 20 and 10 ppm. The least ERR of 83.62 per cent was observed in the control. The ERR was increased from 2.22 to 11.70 per cent over the control due to application of alanine. The present result is in parallel with the findings of Ito and Arai (1965) and Chakrabarthy and Kaliwal (2012), who observed enhanced resistance and lesser susceptibility of the larvae to deadly infectious diseases, increased ERR and cocoon formation.

Irrespective of the concentrations, the application of alanine significantly reduced the fifth instar larval duration. The treatment with $100 \mathrm{ppm}$ registered minimum larval duration of $163.40 \mathrm{hrs}$, which was statistically on par with 200 ppm (163.75 hrs) and $50 \mathrm{ppm}$ (164.50 hrs). These were followed by 20 and 10 ppm concentrations. The fifth instar larval duration of larval batch fed with untreated mulberry leaves lasted for $170.20 \mathrm{hrs}$. These observations can be corroborated with the findings of Mustafa and Elkaraksy (1990), who found that supplementation of glycine, alanine and serine to silkworm significantly decreased the fifth instar larval duration.

\section{b. Cocoon characters}

The oral application of alanine resulted in improvement in cocoon related characters (Table 2). Among different concentrations studied, alanine @ 100 ppm registered highest cocoon weight of 2.02 $\mathrm{g}$ which showed statistical superiority over all other treatments. This was followed by 200 ppm (1.95 g) and 50 ppm (1.90 g) which were statistically on par. The least cocoon weight of $1.61 \mathrm{~g}$ was observed in the larval batch fed with untreated mulberry leaves. Here, the gain in cocoon weight ranged from 6.21 to 25.47 per cent over the control. This result got strengthened with the findings of Chakrabarthy and Kaliwal (2012), who reported that supplementation of amino acids to silkworm increased the cocoon parameters. Further, the present observations are strengthened by findings of Radjabi (2010), who reported that feeding the silkworm with alanine (0.2 $\%)$ fortified mulberry leaves during fourth and fifth instars of silkworm, once in a day led to significant increase in cocoon weight (1.51 g) over the control (1.35 g).

Table 2. Effect of alanine on cocoon characters of $B$. mori

\begin{tabular}{lcccc}
\hline $\begin{array}{c}\text { Concentration } \\
(\mathbf{p p m})\end{array}$ & $\begin{array}{c}\text { Cocoon } \\
\text { weight }(\mathbf{g})\end{array}$ & $\begin{array}{c}\text { Shell } \\
\text { weight }(\mathbf{g})\end{array}$ & $\begin{array}{c}\text { Shell } \\
\text { ratio (\%) }\end{array}$ & $\begin{array}{c}\text { Filament } \\
\text { length }(\mathbf{m})\end{array}$ \\
\hline 10 & 1.71 & 0.34 & 18.18 & 1030 \\
20 & 1.80 & 0.37 & 20.56 & 1054 \\
50 & 1.90 & 0.40 & 20.05 & 1076 \\
100 & 2.02 & 0.45 & 22.28 & 1105 \\
200 & 1.92 & 0.41 & 21.35 & 1082 \\
Water spray & 1.61 & 0.28 & 17.39 & 1005 \\
Control & 1.61 & 0.28 & 17.39 & 1000 \\
SEd & 0.04 & 0.01 & 0.30 & 9.50 \\
CD (P=0.05) & 0.08 & 0.02 & 0.60 & 19.00 \\
\hline
\end{tabular}

Values are mean of three replications and pooled mean of two rearing. 
The highest shell weight and shell ratio of $0.45 \mathrm{~g}$ and 22.18 per cent, respectively, were recorded in $100 \mathrm{ppm}$ of alanine, which was found to be statistically on par with 200 ppm (0.43 g and $22.18 \%$, respectively). Here, the increase in shell weight and shell ratio was 21.43 and 60.71 per cent, respectively. This treatment was followed by 50 ppm (0.40 g and $20.05 \%$, respectively) and 20 ppm (0.37 g and $20.56 \%$, respectively) which did not differ statistically. The lowest shell weight and shell ratio were recorded in the control. Nagarajan and Radha (1990) observed increased cocoon productivity, shell weight and shell ratio by feeding the silkworm with enriched mulberry leaves with tyrosine, phenylalanine and alanine. Significantly maximum shell weight and shell ratio of $0.335 \mathrm{~g}$ and 20.94 per cent, respectively, were observed, when the silkworm larvae were fed by mulberry leaves treated with alanine @ 1 per cent (Radjabi, 2010). This finding falls more or less in line with the present observations.

Filament length is a very important one among the various characters that contribute to higher silk yield. Silk filament length showed great deal of variation among different treatments. The longest filament with the length of $1105 \mathrm{~m}$ was registered in the larval batch treated with 100 ppm alanine followed by 200 and 50 ppm; however, both the concentrations did not have significant difference. The minimum filament length of $1000 \mathrm{~m}$ was observed in the control. The present observation falls well in line with the findings of Nagarajan and Radha (1990), who observed enhanced silk filament length by supplementing tyrosine, phenylalanine and alanine along with mulberry leaves.

\section{III.Influence of serine on larval and cocoon characters}

\section{a. Larval characters}

Feeding the silkworm larvae with mulberry leaves sprayed with serine at varied concentrations elucidated significant improvement on biological characters of larvae (Fig. 3). The highest mature larval weight of $4.00 \mathrm{~g}$ was observed in $100 \mathrm{ppm}$. Here, the weight gain in the mature larval is 18.69 per cent over the control. The next better concentrations were in the order of 50,200, 20 and $10 \mathrm{ppm}$. The least mature larval weight of $3.37 \mathrm{~g}$ was registered in the larval batch fed with untreated mulberry leaves. The present observations can be corroborated with the studies of Vadivel (1995) and Gokul (2015) who reported that the supplementation of amino acids viz., glycine and serine resulted in increased fifth instar larval weight.

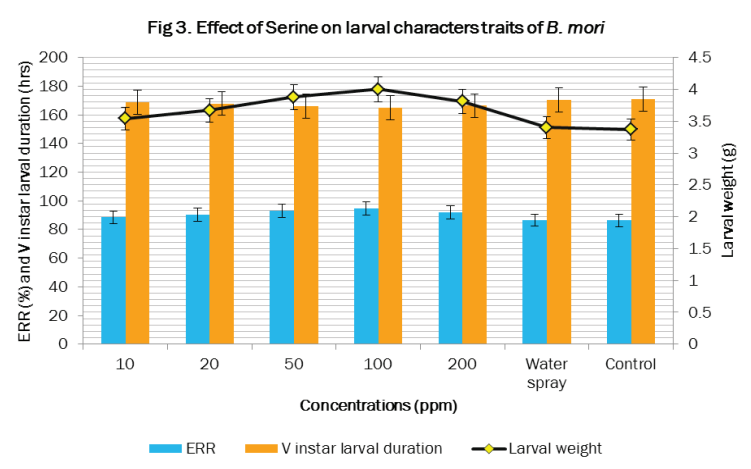

Highest ERR of 94.70 per cent was recorded in $100 \mathrm{ppm}$ which was found to be statistically superior over all other concentrations. This was followed by 50 (92.95\%) and 200 (92.00 \%) ppm, which were found to be on par among themselves. The least ERR of 86.30 per cent was observed in control. This observation falls more or less in line with the findings of Vadivel (1995) who reported that oral application of different amino acids had resulted in increased ERR and other economic traits of silkworm. Ramesh et al. (2018) reported that feeding the B. mori larvae with mulberry leaves treated with 0.25 per cent serine during third, fourth and fifth instars pronounced significant enhancement in ERR (88.95 g) over the control (82.27 g). This finding also supports the present observations.

The least fifth instar larval duration of 165.00 hrs was noted in the larval batch fed by mulberry leaves fortified with $100 \mathrm{ppm}$ serine, which was found to be on par with 50 and 200 ppm. Here, the reduction in fifth larval duration ranged from 1.81 to 3.51 per cent. This result falls in line with Mustafa and Elkaraksy (1990), who reported that supplementation of glycine, alanine and serine significantly decreased the fifth instar larval duration.

\section{b. Cocoon characters}

Studies clearly proved that enrichment of mulberry leaves with Serine in different concentrations showed significant improvement on the cocoon characters of silkworm (Table 3). Highest cocoon weight of $2.05 \mathrm{~g}$ was observed in the larval batch fed by mulberry leaves sprayed with 100 ppm of serine, which showed statistical superiority over all other concentrations. The cocoon weight was increased by 19.88 per cent over the control due to application of amino acid. This treatment was followed by 50 (1.98 g) and 200 (1.95 g) ppm, which were on par with each other. The minimum cocoon weight of $1.71 \mathrm{~g}$ was observed in control. The present observations are in agreement with Gokul (2015), who revealed that oral application of serine @ 100 ppm from first day of fifth instar to spinning; daily once in the morning significantly enhanced the cocoon weight to 
$2.04 \mathrm{~g}$ from $2.00 \mathrm{~g}$ in control. Ramesh et al. (2018) also observed that supplementation of serine @ 0.25 per cent to late-age silkworm significantly increased the cocoon weight by 66.12 per cent. This finding also supports the present observations.

Table 3. Effect of serine on cocoon characters of B. mori

\begin{tabular}{lcccc}
\hline $\begin{array}{c}\text { Concentration } \\
\text { (ppm) }\end{array}$ & $\begin{array}{c}\text { Cocoon } \\
\text { weight }(\mathbf{g})\end{array}$ & $\begin{array}{c}\text { Shell } \\
\text { weight }(\mathbf{g})\end{array}$ & $\begin{array}{c}\text { Shell } \\
\text { ratio (\%) }\end{array}$ & $\begin{array}{c}\text { Filament } \\
\text { length }(\mathbf{m})\end{array}$ \\
\hline 10 & 1.80 & 0.31 & 17.22 & 1026 \\
20 & 1.87 & 0.35 & 18.72 & 1047 \\
50 & 1.98 & 0.40 & 20.20 & 1075 \\
100 & 2.05 & 0.45 & 21.95 & 1100 \\
200 & 1.95 & 0.39 & 20.00 & 1068 \\
Water spray & 1.71 & 0.27 & 15.79 & 1007 \\
Control & 1.71 & 0.27 & 15.79 & 1003 \\
SEd & 0.03 & 0.02 & 0.49 & 9.10 \\
CD (P=0.05) & 0.06 & 0.03 & 1.00 & 18.00 \\
\hline
\end{tabular}

Values are mean of three replications and pooled mean of two rearing.

The administration of serine also improved the shell weight and shell ratio of the cocoon, irrespective of the concentrations. However, maximum shell weight and shell ratio of $0.45 \mathrm{~g}$ and 21.95 per cent, respectively were recorded in $100 \mathrm{ppm}$. Here, the enhancement in shell weight and shell ratio was 29.63 and 12.69 per cent, respectively over the control. The next better treatments were in the order of 50 and 200 ppm, however, both of which did not differ statistically from each other. The least shell weight and shell ratio of $0.27 \mathrm{~g}$ and 15.79 per cent, respectively, were observed in control. Enrichment of mulberry leaves with serine @ 0.25 per cent and feeding during late instars of silkworm significantly improved the shell weight to $0.80 \mathrm{~g}$ from $0.60 \mathrm{~g}$ in control. Likewise, the same concentration also enhanced the shell ratio to 20.02 from 18.07 per cent in control (Ramesh et al., 2018). Further, Gokul (2015) found that fortification of mulberry leaves with $100 \mathrm{ppm}$ serine and feeding the larvae from the beginning of the fifth instar until spinning, once in a day showed superior shell weight $(0.47 \mathrm{~g})$ and shell ratio $(22.08 \%)$ over the control. The present observations got strengthened by the above findings.

The filament length of the cocoon was increased due to per os application of alanine to silkworm larvae. The improvement ranged from 2.29 to 9.67 per cent with maximum silk filament length of $1100 \mathrm{~m}$. The minimum filament length of $1003 \mathrm{~m}$ was registered in the control. Shyamala and Gowda (1980) and, Chakrabarthy and Kaliwal (2012) reported that supplementation of amino acids individually or in combinations increased the silk filament length and raw silk productivity. The supplementation of glycine and serine increased filament length and weight by increasing fibroin and sericin, respectively (Sridhar and Radha, 1986; Krishnapa, 1987; Dai et al., 1987; Asakura et al., 1991). The filament length was significantly enhanced to 1193 m from 1031 m (control) due to the application of serine @ 100 ppm to silkworm (Gokul, 2015). Ramesh et al. (2018) found that feeding the late-age silkworm with mulberry leaves sprayed with 0.25 per cent serine elucidated a significant increase in the length of silk filament from $810 \mathrm{~m}$ (control) to $964 \mathrm{~m}$. The above findings are in parity with the present observations.

\section{CONCLUSION}

It is clear from the studies that supplementation of glycine, alanine and serine at the concentration of 10,100 and 100 ppm, respectively to silkworm larvae once daily in the morning from the first day of the fifth instar to spinning improved the larval growth as well as cocoon characters. Per os administration of amino acids at specific concentrations also significantly decreased the fifth instar larval duration.

\section{Ethics statement}

No specific permits were required for the described field studies because no human or animal subjects were involved in this research.

\section{Consent for publication}

All the authors agreed to publish the content.

\section{Competing interests}

There was no conflict of interest in the publication of this content

\section{Data availability}

All the data of this manuscript are included in the M.S. No separate external data source is required. If anything is required from the M.S., certainly, this will be extended by communicating with the corresponding author through corresponding official mail : murugehka2002@yahoo.co.in

\section{REFERENCES}

Arai, N. and T. Ito. 1964. Amino acid requirements of silkworm, Bombyx mori L. J. Seric. Sci. Japan., 33: 107-110.

Asakura, T., Demura, M., Nagashima, M., Sukaguchi, R., Osanai, M. and K. Ogawa. 1991. Metabolic flux and studied by CNMR in vivo and in vitro. Insect Biochemistry., 21(7): $743-748$.

Beadle, L. C. and I. Shaw. 1950. The retention of salt and the regulation of the non-protein nitrogen fraction in the blood of the aquatic larva Sialis lutaria. Journal of Experimental Biology., 27: 96-109.

Chakrabarthy, S. and B. B. Kaliwal. 2012. Application of arginine, histidine and their mixture on economic traits of the silkworm, Bombyx mori L. DAV International Journal of Science., 1(2): 107-111. 
Chen, P. S. and B. Widmer. 1968. Content and synthesis of $r$-aminobutyric acid in the larval brain of Drosophila melanogaster. Experiments., 24: 516-517.

Dai, Y. J., Yao, X. and H. Z. Xia. 1987. Moulting hormone and silk protein synthesis. Effect of ecdysone on the incorporation activity of $\mathrm{H}$-glycine into proteins in the silk gland of the silkworm, Bombyx mori $\mathrm{L}$. Acta Entomologia., 30(3): 233-238.

Dandin, S. B. and K. Gridhar. 2014. Handbook of Sericulture Technologies. Central Silk Board, Bangalore, pp.315-325.

Gokul, M. 2015. Effect of exogenous supplementation of amino acids and minerals on economic traits of silkworm, Bombyx mori L. M.Sc. (Seri.) Thesis, Tamil Nadu Agricultural University, Coimbatore, p. 101.

Inokuchi, T., Horie, Y. and T. Ito. 1967. Nutrition of the silkworm, Bombyx mori - XIX. Effects of omission of essential aminoacids in each of the second to the fifth instars. Bulletin Sericulture Experimental Station Japan., pp.612-635.

Ito, T. 1967. Nutritional requirements of the silkworm Bombyx mori L. Proceedings Japan Academy., 43: 57-61.

Ito, T. and N. Arai. 1965. Nutrition of the silkworm, Bombyx mori L. Requirements for aspartic and glutamic acids. Journal of Insect Physiology., 12: 861-869.

Ito, T. and N. Arai. 1967. Nutritive effects of alanine, cysteine, glycine, serine and tyrosine on the silkworm, Bombyx mori L. Journal of Insect Physiology., 13: 1813-1824.

Kabila, V., Subburathinam, K. M. and J. Sulochanachetty. 1994. Growth and economic characters of silkworm, Bombyx mori L. on feed enriched with neutralized aspartic acid. Indian Journal of Sericulture., 33(1): 80-81.

Kodie, F., Nagayama, H. and K. Shimura. 1965. Transaminases in silkworm tissues (In: Japanese with English Summary). Journal of Agriculture and Chemical Society of Japan., 29: 987-990.

Krishnapa, J. B. 1987. Influence of amino acids supplementation on growth and development of mulberry silkworm, Bombyx mori L. M.Sc. Dissertation, University of Agri. Sciences, Bangalore, p.13.

Mustafa, S. M and I. A. Elkaraksy. 1990. The effect of certain aminoacids on silkworm, Bombyx mori. L. Agricultural Research Review., 66(1): 105-109.
Nagarajan, P. and N. V. Radha. 1990. Supplementation of aminoacids through mulberry leaf for increased silk production. Indian Silk., 29(4): 21-22.

Panse, V. G. and K. Sukhatme. 1957. Statistical Methods for Agricultural Workers, ICAR, New Delhi. p.328.

Radjabi, R., Ebadi, R., Mirhoseini, S. Z. and S. Nair. 2010. Effect of leaves enrichment with amino acid supplementary nutrients on Bombyx mori L. Acad. J. of Ent., 3(1): 45-51.

Ramesh, V., Pushparaj, K., Prabu, G. P. and P. Rajasekar. 2018. Nutritional supplementation of amino acid L-serine on silkworm, Bombyx mori L. Iarvae in relation to growth rate and silk production. Research Journal of Life Sciences, Bioinformatics, Pharmaceutical and Chemical Sciences., 4(5): $301-312$

Ravi, K. N., Shankar, K. A., Puttaraju, T. B. and B. Puttasamy. 1994. Effect of feeds supplementation on silkworm growth, cocoon weight and silk quality. Second National Symposium on prospects and problems of sericulture in India. Dept. of Zoology. Madras University. Postgraduate Extension Centre, Vellore, Tamil Nadu, p.63-66.

Saad, M. S. I., Helaly, W. M. M. and E. A. E. Sheikh. 2019. Biological and physiological effects of pyriproxyfen insecticide and amino acid glycine on silkworm, Bombyx mori L. Bulletin of the National Research Centre., 43:145-151.

Sengupta, K., B. D. Singh and J. C. Mustafi. 1972. Nutrition of silkworm Bombyx mori L. Studies on the enrichment of mulberry leaf with various sugars, proteins, amino acids and vitamins for vigorous growth of worms and increased cocoon crop production. Indian Journal of Sericulture., 11: 11-19.

Sharada, K. and J. V. Bhat. 1956. Effect of chloromycetin and glycine on the growth and reproduction of silkworm, Bombyx mori L. Journal of Indian Institute of Sciences., 38: 196-197.

Shyamala, M. B. and B. L. V. Gowda. 1980. Effect of supplementing phenylalanine to mysore- 5 and local varieties of mulberry fed to silkworm, Bombyx mori L. Proceedings of Sericulture Symposium, Coimbatore, pp.88-92.

Sridhar, P. and N. V. Radha. 1986. Effect of supplementing glycine to the feed of silkworm, Bombyx mori L. Indian Silk., 27(1): 115-121.

Vadivel, K. 1995. Effect of supplementation of amino acids on economic characters of silkworm, Bombyx mori L. M.Sc. Thesis, Tamil Nadu Agricultural University, Coimbatore, pp.32-49. 